\title{
Measles in Afghan refugees: Challenges, efforts and recommendations
}

\section{Zarmina Islam ${ }^{\mathrm{a}}$, Zoaib Habib Tharwani ${ }^{\mathrm{b}}$, Malaika Saeed Butt ${ }^{\mathrm{c}}$, Sean Kaisser Shaeen ${ }^{\mathrm{a}}$, Muhammad Saad Arshad ${ }^{\mathrm{b}}$, Muhammad Abdullah Khalid ${ }^{\mathrm{a}}$, Mohammad Yasir Essar ${ }^{\mathrm{d}, *}$, Shoaib Ahmad ${ }^{\mathrm{e}}$, Helen Onyeaka ${ }^{\mathrm{f}}$}

${ }^{a}$ Faculty of Medicine, Dow Medical College, Dow University of Health Sciences, Pakistan

${ }^{\mathrm{b}}$ Dow University of Health Sciences (Dow Medical College), Pakistan

${ }^{\mathrm{c}}$ Dow Medical College, Dow University of Health Sciences, Pakistan

${ }^{\mathrm{d}}$ Kabul University of Medical Sciences, Kabul, Afghanistan

e District Head Quarters Teaching Hospital, Faisalabad, Pakistan

${ }^{\mathrm{f}}$ University of Birmingham, UK

\section{A R T I C L E I N F O}

\section{Keywords}

Afghanistan

Measles

MMR Vaccine

COVID-19 pandemic

Coronavirus

Humanitarian crisis

Measles eradication

\begin{abstract}
A B S T R A C T
Recently, measles outbreaks have been reported across Afghanistan, and in many refugees. Although, Afghanistan has a previous history of measles outbreaks, the presence of such epidemics during a humanitarian crisis is burdening a fragile healthcare system. In addition, it is creating new challenges for Afghan refugees who are endangered by political conditions of the country. Despite efforts such as vaccination to reduce the number of cases in Afghanistan, there are still multiple outbreaks. Various factors such as political conflict, insecurity, internal displacement, supply chain issues and, most recently, COVID-19 have hampered the eradication of measles. High mortality rate, faster transmission, and clinical similarities with COVID-19 are exacerbating challenges for refugees, who are now facing delays in resettlement, especially in the United States. To curb the spread of measles, refugees need immediate and effective vaccination measures, and access to healthcare information in their own languages.
\end{abstract}

\section{Introduction}

Measles, also called rubeola, is a viral infection which mostly affects children under the age of five. ${ }^{1}$ Signs and symptoms appear 10-14 days after being infected and include fever, dry cough, runny nose, sore throat, conjunctivitis, Koplik's spots, and skin rashes. ${ }^{1}$ Although, high vaccination rates have decreased the prevalence of measles deaths by $78 \%$ between 2000 and 2008 worldwide. ${ }^{1,2}$ In 2019, 870,000 cases of measles were reported, and 208,000 deaths globally. In Africa, measles cases rose by $700 \%$ between 2018 and 2019. ${ }^{3,4}$ Similarly, Pakistan and India are also currently facing a measles outbreak. ${ }^{5}$ Due to the pandemic, routine immunization was limited by supply and cold chain issues, reduced healthcare capacity, and a primary focus on managing COVID-19 cases. $^{3}$

Historically, measles outbreaks have also plagued Afghanistan. The protracted war and conflicts with different political groups may have also limited the immunization of measles. For example, in 2012, $80 \%$ of all outbreaks in the country were measles, primarily affecting children under the age of $10^{2}$. In the February 2012 outbreak 3,600 people were also vaccinated. ${ }^{6}$ The Ministry of Public Health and World Health Organization (WHO) reported 20 confirmed children deaths from measles and pneumonia in provinces of Ghor and Baghdis. ${ }^{7}$ The outbreak initially affected the villages of Lafra valley, and the most affected district was Cheghchran district, a part of Gohr province, but WHO took immediate measures by setting up temporary clinics to contain the outbreak. $^{7}$

In May 2021 WHO reported 741 cases with Paktika province being most affected accounting for 131 reported cases. ${ }^{8}$ Whereas, Shangla district of Khyber Pakhtunkhwa (KPK), province of Pakistan that shares border with Afghanistan, has also reported above 400 patients with measles. ${ }^{5}$ Recently, Center for Disease Control (CDC) reported 16 new measles cases among Afghan refugees, and since measles is highly

\footnotetext{
* Corresponding author.

E-mail addresses: zarmina2001@gmail.com (Z. Islam), zoaibhabib@hotmail.com (Z.H. Tharwani), malaikasaeedbutt@hotmail.com (M.S. Butt),drseanks@gmail.

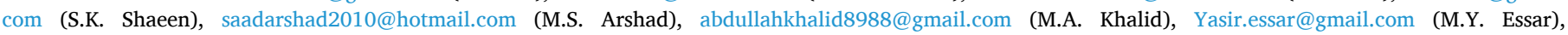
shoaibahmad442@gmail.com (S. Ahmad), h.onyeaka@bham.ac.uk (H. Onyeaka).
} 
contagious and affects around 9 out of 10 unprotected people who come into contact, this proposes a threat to healthcare systems globally. ${ }^{9}$ Out of 70 Afghan refugees who were sent to India, 16 were tested positive for COVID-19 (4). Since 2019, Afghanistan has a 12-month measles incidence of $5-10$ cases per 100,000 people. ${ }^{10}$ Higher transmission is a threat globally as refugees escape Afghanistan to resettle in countries such as the United States (US), however lack of immunization have delayed evacuation efforts.

The withdrawal of the United States and its allies has brought forth numerous challenges to the country, particularly, the healthcare system. The challenges associated with the healthcare system are lack of financial support, lack of staff in the hospital, and lack of supplies in the hospitals. ${ }^{11}$ As a result of the humanitarian crisis, increased evacuation efforts have exacerbated both the spread of COVID-19 and measles. Thus, this paper aims to comment on the current challenges associated with measles in Afghan refugees and make recommendations.

\section{Background}

As Afghanistan currently ranks 6th in the world for the number of reported measles cases, a previous history of outbreaks poses a threat to its weak healthcare infrastructure. ${ }^{12}$

Afghanistan has made great progress in its efforts to eradicate measles with a reported vaccine coverage of $79 \%$ in 2010 from $42 \%$ in 2000 which has been attributed to the mass campaign of 2001-2002. ${ }^{2}$ However, a seasonal relationship has been observed for measles outbreaks in Afghanistan with an epidemic peak wave occurring every 7 years in similar conflict affected provinces. ${ }^{2}$ This has yielded multiple outbreaks in the past; although, a progressive rise in measles may also be due to improved surveillance measures. ${ }^{2}$

Currently, the country's main healthcare service provider "Sehatmandi" is not functioning properly, which will result in the resurgence of the many infectious diseases including measles. Only $17 \%$ of all Sehatmandi, a donor support, healthcare facilities are functional which has affected basic and emergency health care, polio eradication program, and COVID-19 vaccination programs. ${ }^{6}$ As such, Afghanistan does not have enough resources to establish a proper healthcare system that can keep up with the prevention and surveillance of both measles and COVID-19.

As of now there's a lack of essentials such as Personal Protective Equipment (PPEs) with only enough to accommodate $5 \%$ of total confirmed cases. Alongside, there is a shortage of healthcare professionals (HCPs); a ratio of 9.4 HCPs and 1.9 physicians per 10000 individuals. ${ }^{13}$ According to the Ministry of Public Health of Afghanistan they are only able to test 2000 out of 10000 to 20000 samples received daily for COVID-19. Thus, this leads to underreporting of real time cases which poses a security risk for refugees that need to leave immediately. ${ }^{13}$ Moreover, those with measles will be unable to receive timely healthcare services, especially in areas with high conflict, and poor availability of healthcare services such as rural areas. For refugees stuck in other countries, delays in refugee claim processing further adds to their stress, considering a high number of women and children among these refugees. ${ }^{14}$

Political instability has resulted in a humanitarian crisis and multiple instances of civil unrest, which are burdening an already failing healthcare system. This includes closure of $87 \%$ of the most essential health centers in the country. ${ }^{15}$ In addition, internal displacement has increased mobility promoting the spread of measles, whereas prior socioeconomic disparities, and political climate have complicated access to basic healthcare services. ${ }^{16,17}$ Globally 117 million children might miss out on measles, mumps, and rubella (MMR) vaccine due to COVID-19. ${ }^{10}$ The WHO states a possible reason for the decrease in MMR vaccinations could also be due to a progressive rise of insecurity. As such, an inverse relationship between the plummeting vaccination rates and the increasing measles cases have burdened refugees, who are now finding greater challenges to becoming refugees in other nations.
However, the pandemic is another overwhelming factor to consider. As of January 2022, 159,380 COVID-19 cases have been reported in Afghanistan with the highest daily average reported on June 22, 2021, corresponding to the rise in delta variant. ${ }^{18}$ Whereas, only around $6.6 \%$ of Afghanistan's population has received the first dose for COVID-19 vaccination. ${ }^{18}$ Furthermore, the overlapping clinical manifestations of COVID-19 and measles are complicating diagnosis since an early differential diagnosis is key to select the optimal treatment to help reduce the rate of mortality. ${ }^{2,19}$ The potential threat of co-infection between measles and COVID-19, as well as misdiagnosis may further complicate the refugee claimant process, especially for those wanting to resettle in the US. Lastly, both COVID-19 and measles have an asymptomatic latency period where they are susceptible to spreading infections 4 days before and after the occurrence of the rash with symptomatic phase being most infective. ${ }^{4}$ Hence, screening for measles is particularly crucial for the Afghan refugees as multiple outbreaks evidenced to outbreaks in similar high-density population areas leading to a high mortality rate of up to $34 \% .^{20}$

Taken together, those attempting to access vaccination centers, or treatment are largely limited by pre-existing political, and socioeconomic conditions in the country.

\section{Efforts and recommendations}

In October 2021, 9,139 Afghan refugees stuck at US military bases have been vaccinated. ${ }^{21}$ The CDC has implemented a mandatory measles vaccination and 21-day quarantine in place for Afghan refugees. Refugees are required to follow appropriate health protocols prolonging the refugee resettlement process and creating barriers for those who have not evacuated. ${ }^{22}$

Recommendations for those taking in Afghan refugees start with first medically screening the refugees at "Safe Haven" sites, such as foreign military bases, that house them prior to resettlement in their host country. Many countries including the US, are housing refugees in various countries temporarily as a means of processing and preparing for the upcoming influx of refugees. ${ }^{14}$ It is at these sites where the refugees are being medically screened for COVID-19 and should be continued to be used to simultaneously screen for measles. Secondly, it is imperative to educate the public at these sites including the host and the refugees themselves in their respective common languages, specifically Pashto and Dari, on the differences between the signs and symptoms of measles and COVID-19 patients in such a way that they are not confused between the two and such that they can seek appropriate medical help promptly. The CDC already has provided pamphlets for the refugees in various languages, including those previously mentioned, that can prove helpful in the educational process for various countries preparing to take in Afghan refugees across the board. ${ }^{22}$ Furthermore, these pamphlets can be supplemented by guiding the religious community leaders and elders of the refugees into informing and encouraging other refugees to learn the signs and symptoms of COVID-19 and measles as well to seek medical help when warranted. Moreover, further research is warranted for finding the prevalence of measles, knowledge, attitude, and practice towards measles among Afghan refugees, especially in countries where refugees are temporarily staying. Also, areas in Afghanistan with higher geographic prevalence of measles need to be identified through collaborative work with Taliban officials to better establish prevention protocols. The Taliban should ensure the WHO and other international organizations on the ground that a safer outreach of health services to areas with high prevalence of measles will be warranted.

\section{Conclusion}

Concurrent rise in COVID-19 cases, poor healthcare capacity in Afghanistan, higher transmission of measles because of internal displacement, and current evacuation efforts predisposes Afghanistan and other nations to measles outbreaks. Additional barriers to becoming 
refugees complicate and delay evacuation efforts, possibly endangering the security of Afghan refugees. Given the situation, Afghan refugees need immediate international health aid to increase vaccination and awareness efforts.

\section{Funding}

Not applicable.

\section{Authors' contributions}

Zarmina Islam conceived the idea and design, wrote the conclusion, abstract, and edited the revised draft and organized references; Zoaib Habib Tharwani wrote the introduction; Malaika Saeed Butt wrote the discussion; Sean Kaisser Shaeen wrote the recommendations; Muhammad Saad Arshad wrote the efforts; Muhammad Abdullah Khalid wrote the discussion; Mohammad Yasir Essar, Shoaib Ahmad, and Helen Onyeaka made the critical comments and revision. All authors revised and approved the final draft.

\section{Declaration of competing interest}

The authors declare that they have no competing interests.

\section{Acknowledgements}

None.

\begin{tabular}{|c|c|}
\hline bbreviat & tions \\
\hline VID-19 & Coronavirus disease \\
\hline MMR & Mumps, Measles, Rubella \\
\hline $\mathrm{CDC}$ & Center for Disease Control \\
\hline $\mathrm{PP}$ & Healthcare Professionals \\
\hline
\end{tabular}

\section{References}

1 Tannous LK, Barlow G, Metcalfe NH. A short clinical review of vaccination against measles. JRSM Open. 2014;5(4):1-6. https://doi.org/10.1177/2054270414523408.

2 Operational guideline for Measles epidemic response in Afghanistan | HumanitarianResponse. https://www.humanitarianresponse.info/en/operations/af ghanistan/document/operational-guideline-measles-epidemic-response-afghanistan. Accessed November 13, 2021.

3 Measles. MSF medical response. https://www.msf.org/measles. Accessed November 13, 2021.

4 Uwishema O, Adriano LF, Torbati T, Onyeaka H. Measles crisis in Africa amidst the COVID-19 pandemic: delayed measles vaccine administration may cause a measles outbreak in Africa. J Med Virol. 2021;93(10):5697-5699. https://doi.org/10.1002/ JMV.27150.
5 Pakistan: measles outbreak in Shangla district, KP - outbreak news today. http://out breaknewstoday.com/pakistan-measles-outbreak-in-shangla-district-kp-82615/. Accessed November 12, 2021.

616 Afghan evacuees test Covid positive, sent for quarantine - coronavirus Outbreak News. https://www.indiatoday.in/coronavirus-outbreak/story/afghan-evacuees-in dia-covid-positive-1844944-2021-08-25. Accessed November 12, 2021.

7 Afghanistan Health Alert: Measles outbreak - Afghanistan | ReliefWeb. Accessed November 12, 2021. https://reliefweb.int/report/afghanistan/afghanistan-healthalert-measles-outbreak.

8 WHO EMRO. Weekly epidemiological monitor | information resources | epidemic and pandemic diseases. http://www.emro.who.int/pandemic-epidemic-diseases/in formation-resources/weekly-epidemiological-monitor.html. Accessed November 12, 2021.

9 Afghanistan evacuees must quarantine after measles and mumps are detected | Everyday Health. Accessed November 12, 2021. https://www.everydayhealth. com/infectious-diseases/afghanistan-evacuees-must-quarantine-after-measles-andmumps-are-detected/.

10 More than 117 million children at risk of missing out on measles vaccines, as COVID19 surges - Measles \& Rubella Initiative. Accessed November 12, 2021. https://meas lesrubellainitiative.org/measles-news/more-than-117-million-children-at-risk-ofmissing-out-on-measles-vaccines-as-covid-19-surges/.

11 Narain K, Rackimuthu S, Essar MY, Vink M. Call for solidarity: the war may be over in Afghanistan but the health crises continue. J Glob Health. 2022;12, 03002.

12 Global measles outbreaks. https://www.cdc.gov/globalhealth/measles/data/global -measles-outbreaks.html. Accessed November 12, 2021.

13 Acute health needs in Afghanistan must be urgently addressed and health gains protected. https://www.who.int/news/item/22-09-2021-acute-health-needs-in-a fghanistan-must-be-urgently-addressed-and-health-gains-protected. Accessed November 12, 2021.

14 Ramstein Air Base: The Kabul airlift has ended, but a US operation to get 14,000 people off a German base is far from over - CNN. Accessed November 12, 2021. https: //edition.cnn.com/2021/09/01/europe/ramstein-air-base-afghan-evacuees-intlcmd/index.html.

15 Afghanistan: Health system collapse will result in thousands of additional child deaths each month - save the Children | Save the Children International. Accessed November 1, 2021. https://www.savethechildren.net/news/afghanistan-health-s ystem-collapse-will-result-thousands-additional-child-deaths-each-month.

16 Shah J, Essar MY, Qaderi S, et al. Respiratory health and critical care concerns in Afghanistan. Lancet Respir Med. 2021. https://doi.org/10.1016/S2213-2600(21) 00583-x.

17 Essar MY, Hasan MM, Islam Z, Riaz MMA, Aborode AT, Ahmad S. COVID-19 and multiple crises in Afghanistan: an urgent battle. Conflict Health. 2021;15(1):1-3. https://doi.org/10.1186/S13031-021-00406-0/METRICS.

18 Afghanistan: the latest coronavirus counts, charts and maps. https://graphics.reuters .com/world-coronavirus-tracker-and-maps/countries-and-territories/afghanistan/. Accessed November 12, 2021.

19 Rana MS, Usman M, Alam MM, Ikram A, Salman M. Overlapping clinical manifestations of COVID-19 with endemic infectious diseases in Pakistan: a looming threat of multiple lethal combinations. Infect Ecol Epidemiol. 2021;11:1. https://doi. org/10.1080/20008686.2021.1873494. https://doi.org/10.1080/2000868620 211873494.

20 HAN Archive. 00452 | health alert network (HAN). https://emergency.cdc.gov/han/ 2021/han00452.asp. Accessed November 12, 2021.

21 Thousands of Afghan refugees stuck at US bases in Germany - InfoMigrants. Accessed November 12, 2021. https://www.infomigrants.net/en/post/35514/thousands-of-a fghan-refugees-stuck-at-us-bases-in-germany.

22 CDC's response to Afghan evacuees | CDC. Accessed November 12, 2021. htt ps://www.cdc.gov/afghan-evac/index.html. 